University of Nebraska - Lincoln

DigitalCommons@University of Nebraska - Lincoln

Timothy J. Gay Publications

Research Papers in Physics and Astronomy

October 1992

\title{
State-selective capture in collisions of protons with noble gases
}

M. Schulz

University of Missouri-Rolla, Rolla, Missouri

D. M. Blakenship

University of Missouri-Rolla, Rolla, Missouri

S. W. Bross

University of Missouri-Rolla, Rolla, Missouri

A. D. Gaus

University of Missouri-Rolla, Rolla, Missouri

Timothy J. Gay

University of Nebraska - Lincoln, tgay1@unl.edu

See next page for additional authors

Follow this and additional works at: https://digitalcommons.unl.edu/physicsgay

Part of the Physics Commons

Schulz, M. ; Blakenship, D. M.; Bross, S. W.; Gaus, A. D.; Gay, Timothy J. ; Htwe, W.; Park, J. T.; and Peacher, J. L., "State-selective capture in collisions of protons with noble gases" (1992). Timothy J. Gay Publications. 19.

https://digitalcommons.unl.edu/physicsgay/19

This Article is brought to you for free and open access by the Research Papers in Physics and Astronomy at DigitalCommons@University of Nebraska - Lincoln. It has been accepted for inclusion in Timothy J. Gay Publications by an authorized administrator of DigitalCommons@University of Nebraska - Lincoln. 
Authors

M. Schulz, D. M. Blakenship, S. W. Bross, A. D. Gaus, Timothy J. Gay, W. Htwe, J. T. Park, and J. L. Peacher 
Phys. Rev. A 46, 3870 - 3876 (1992)

[Issue 7 - October 1992]

\section{State-selective capture in collisions of protons with noble gases}

M. Schulz, D. M. Blankenship, S. W. Bross, A. D. Gaus, T. J. Gay, W. Htwe, J. T. Park, and J. L. Peacher

Department of Physics, University of Missouri-Rolla, Rolla, Missouri 65401

Laboratory for Atomic and Molecular Research, University of Missouri-Rolla, Rolla, Missouri 65401

Received 8 April 1992

We have measured coincidences between neutral $\mathrm{H}$ atoms and Lyman- $\alpha$ photons for collisions between $50-\mathrm{keV}$ protons and noble gases as a function of the projectile scattering angle. The coincidences are dominated by capture to the $2 p$ state of the projectile. While the total cross sections depend strongly on the target, the shape of the angular distribution of the differential cross sections was found to depend only weakly on that parameter. The data indicate that electrons are captured predominantly from the outermost shell of the target atom for the collision systems studied here.

(C)1992 The American Physical Society

URL: http://link.aps.org/abstract/PRA/v46/p3870

DOI: 10.1103/PhysRevA.46.3870

PACS: $34.70 .+\mathrm{e}, 34.50 . \mathrm{Fa}$ 


\title{
State-selective capture in collisions of protons with noble gases
}

\author{
M. Schulz, D. M. Blankenship, S. W. Bross, A. D. Gaus, T. J. Gay, W. Htwe, J. T. Park, and J. L. Peacher \\ Department of Physics, University of Missouri-Rolla, Rolla, Missouri 65401 \\ and Laboratory for Atomic and Molecular Research, University of Missouri-Rolla, Rolla, Missouri 65401
}

(Received 8 April 1992)

\begin{abstract}
We have measured coincidences between neutral $\mathrm{H}$ atoms and Lyman- $\alpha$ photons for collisions between $50-\mathrm{keV}$ protons and noble gases as a function of the projectile scattering angle. The coincidences are dominated by capture to the $2 p$ state of the projectile. While the total cross sections depend strongly on the target, the shape of the angular distribution of the differential cross sections was found to depend only weakly on that parameter. The data indicate that electrons are captured predominantly from the outermost shell of the target atom for the collision systems studied here.
\end{abstract}

PACS number(s): 34.70. $+\mathrm{e}, 34.50 . \mathrm{Fa}$

\section{INTRODUCTION}

Electron capture in ion-atom collisions has been studied extensively since the first measurements in the 1920s [1-7]. Investigations have been performed both experimentally and theoretically for a large variety of collision systems and over a large range of collision energies. In spite of these continuous efforts our understanding of capture processes is still rather incomplete. One reason for this limited understanding is the fact that in a capture process, in contrast to an excitation process, for example, the nucleus with which the electron is associated changes, as does the electron's subsequent average linear momentum. The correct description of this change is a difficult task, involving the introduction of electron translational factors (see, e.g., Refs. [8-10]). Therefore, it is important to measure capture cross sections for many collision systems over a large range of projectile energies, scattering angles, and initial and final electron states so that detailed tests of theoretical calculations can be made.

Total capture cross sections have been measured at projectile energies ranging from as low as $0.1 \mathrm{eV} / \mathrm{amu}$ [11] for highly charged ions to as high as a few $\mathrm{MeV} / \mathrm{amu}$ [e.g., [12]). The agreement between experimental data and theoretical calculations varies depending on the projectile energy region and the model used in the calculation. However, there is no theoretical model available at this time which gives good agreement with measured data over a large range of projectile energies. Stateselective capture has been studied especially at small collision energies using energy-gain spectroscopy (see, e.g., Refs. $[13,14])$. At intermediate projectile energies stateselective capture has been studied by detecting photons emitted after capture to an excited state in coincidence with the projectile which has captured one or more electrons $[15,16]$. These experiments provided a better understanding of the $n$ - and $l$-state distribution of the captured electron. A large variety of data exist for differential cross sections for capture to all states (see, e.g., Refs. [17-20]) providing more sensitive tests of theoretical calculations than total cross sections.

Experimental studies of differential cross sections with state-selective capture are still relatively rare. At low projectile energies differential cross sections were measured for capture to the $2 p$ state of hydrogen for protons colliding with $\mathrm{He}$ [21] and Ar [22]. Furthermore, the polarization of Lyman- $\alpha$ radiation following capture to the $2 p$ state in low-energy collisions of protons with $\mathrm{He}$ was measured as a function of scattering angle [23]. However, in this latter work no absolute cross sections were quoted. We have recently reported measured differential cross sections for capture to the $2 p$ state of hydrogen for protons colliding with $\mathrm{He}$ at intermediate energies [24]. In this work we have extended these studies to investigate the heavier noble-gas targets $\mathrm{Ne}$ and Ar.

\section{EXPERIMENTAL METHOD}

The experimental apparatus is shown schematically in Fig. 1. The experiment was performed with the 200-kV accelerator which is part of the University of

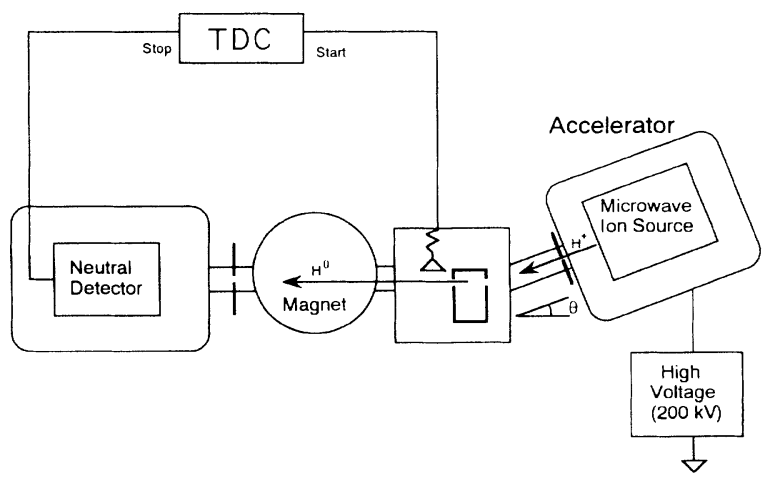

FIG. 1. Experimental setup. The scattering angle of the projectiles was set by rotating the accelerator around the center of the target chamber. The photons emitted by the projectiles after capture to the $2 p$ state were detected by a channeltron detector mounted downstream from the target cell in order to avoid detection of photons emitted by the target atoms. The neutralized projectiles were measured in coincidence with the photons. 
Missouri-Rolla ion energy-loss spectrometer [25]. Protons were produced with a recently developed $2.45-\mathrm{GHz}$ microwave ion source which is similar to one described earlier [26], and accelerated to an energy of $50 \mathrm{keV}$ before being focused into a gas cell. A movable slit in front of the target chamber was used to collimate the beam to a size of approximately $0.15 \times 0.15 \mathrm{~mm}^{2}$. The beam intensity going through the target cell was typically $0.5 \mathrm{nA}$. Protons were separated from the neutralized projectiles magnetically. The $\mathrm{H}$ atoms passed through the $0^{\circ}$ port of the separation magnet and were detected by a focused mesh electron multiplier. A movable slit in front of this detector was used to define the beam trajectory. The scattering angle was set by rotating the accelerator around the center of the gas cell [27]. The accelerator angle could be set with an accuracy of $3.3 \mu \mathrm{rad}$. However, the angular resolution of particles detected with the neutral detector is determined by the geometry of the trajectory-defining slits. From the measured angular distribution of the incident beam, which was determined with the target gas removed from the scattering chamber and the switching magnet turned off, we estimate that our experiment has an angular resolution of approximately $0.2 \mathrm{mrad}$ (see Fig. 2).

The experiment was performed with $\mathrm{He}, \mathrm{Ne}$, and $\mathrm{Ar}$ gas targets. The target region was differentially pumped and had an interaction length of about $1 \mathrm{~cm}$. Target pressures were typically $4 \mathrm{mTorr}$ for Ar, $10 \mathrm{mTorr}$ for $\mathrm{Ne}$, and $50 \mathrm{mTorr}$ for $\mathrm{He}$. The pressure dependence of the neutral-atom count rate was linear at these pressures ensuring single-collision conditions. They were monitored by a capacitance manometer and kept constant to within $5 \%$ by a microprocessor-based pressure controller [28]. Pressures below $2 \times 10^{-6}$ Torr in the surrounding vacuum and a few times $10^{-7}$ Torr in the rest of the beam line could be maintained with these target pressures.

Emitted photons resulting from the collision were detected by a channeltron detector which was mounted downstream from the target cell. With this geometry, photons emitted by the target atoms were not detected. The entrance of the channeltron was covered with a 1-

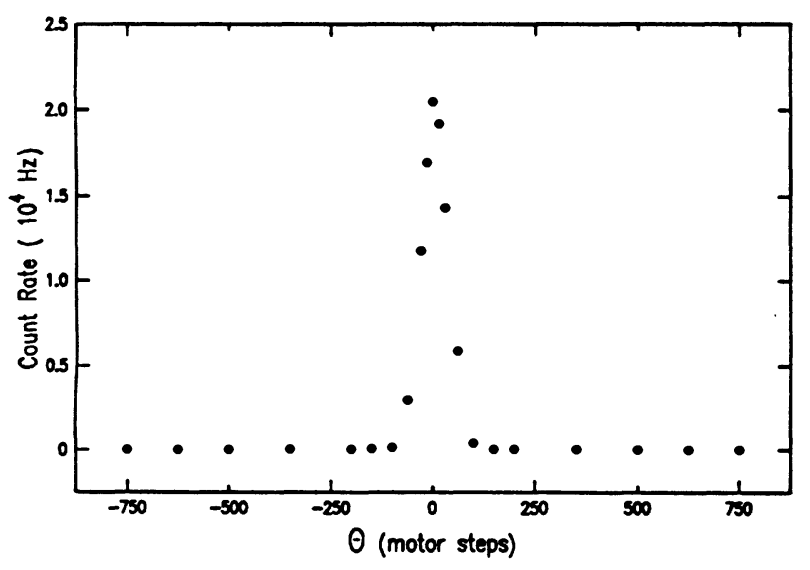

FIG. 2. Angular distribution of the incident proton beam with no target gas. One motor step corresponds to $3.3 \mu \mathrm{rad}$. mm-thick $\mathrm{MgF}_{2}$ window. This kept electrons from being detected and also discriminated against photons with wavelengths shorter than $1140 \AA$ due to absorption in the $\mathrm{MgF}_{2}$ window. Photons with wavelengths longer than $\approx 1400 \AA$ were discriminated against due to the lowenergy cutoff of the channeltron response. Therefore, the photon detectors were sensitive to $\mathrm{H}$ Lyman- $\alpha$ photons $(\lambda=1216 \AA)$, but not to Lyman- $\beta$ photons $(\lambda=1026 \AA)$ or transitions from higher- $n$ states.

The neutral-atom- and photon-detector signals were measured in coincidence. The signal from the photon detector provided the start signal for a time-to-digital converter (TDC) and the neutral-atom detector provided the stop signal. The TDC signals and the total count rates of both detectors were recorded with a computeraided measurement-and-control based data-acquisition system. The total photon count rate was used to normalize the coincidence count rate (see Sec. III). Typically data were taken for five scattering angles in each run. The collection time could be varied for each angle. For small angles the collection time was typically $20 \mathrm{sec}$ in each scan and a few minutes for large angles.

Before and after each run the angular distribution of the total neutral-atom rate (without the coincidence requirement) and of the incident beam was measured. The coincidence count rates as a function of scattering angle were later corrected for the angular spread of the incident beam (see Sec. III).

\section{DATA ANALYSIS}

In Fig. 3 we show a coincidence time spectrum for 50$\mathrm{keV}$ protons colliding with $\mathrm{Ar}$ at an accelerator angle of 0.1 mrad. A sharp coincidence peak at about 700 nsec with a full width at half maximum of approximately 10 nsec can be observed on top of a flat random background. This width we mainly attribute to the uncertainty of the projectile's time of flight due to accelerator-voltage fluctuations and to the charge-collecting time in the detectors. The true coincidences are dominated by capture to the $2 p$ state. Capture to higher-lying states is negligible because of the long transition time for the cascade transition that would have to precede the emission of a Lyman- $\alpha$ photon.

The number of true coincidences were normalized to the total photon rate at each angle. The angular distribution of these normalized coincidence rates is affected by the divergence of the incident beam and were therefore deconvoluted with the measured incident beam profile. This was important because scattering angles smaller than the beam divergence were studied. The deconvolution procedure has been described in detail by Park et al. [27].

In order to obtain absolute differential cross sections the coincidence count rates normalized to the total photon count rates were integrated over the scattering angle and then normalized to the total cross sections for capture to the $2 p$ state. For He the total $2 p$ capture cross sections were taken from a fit to measured data reported previously for a number of projectile energies by different authors [29]. In the case of $\mathrm{Ne}$ and $\mathrm{Ar}$ the total $2 p$ cap- 


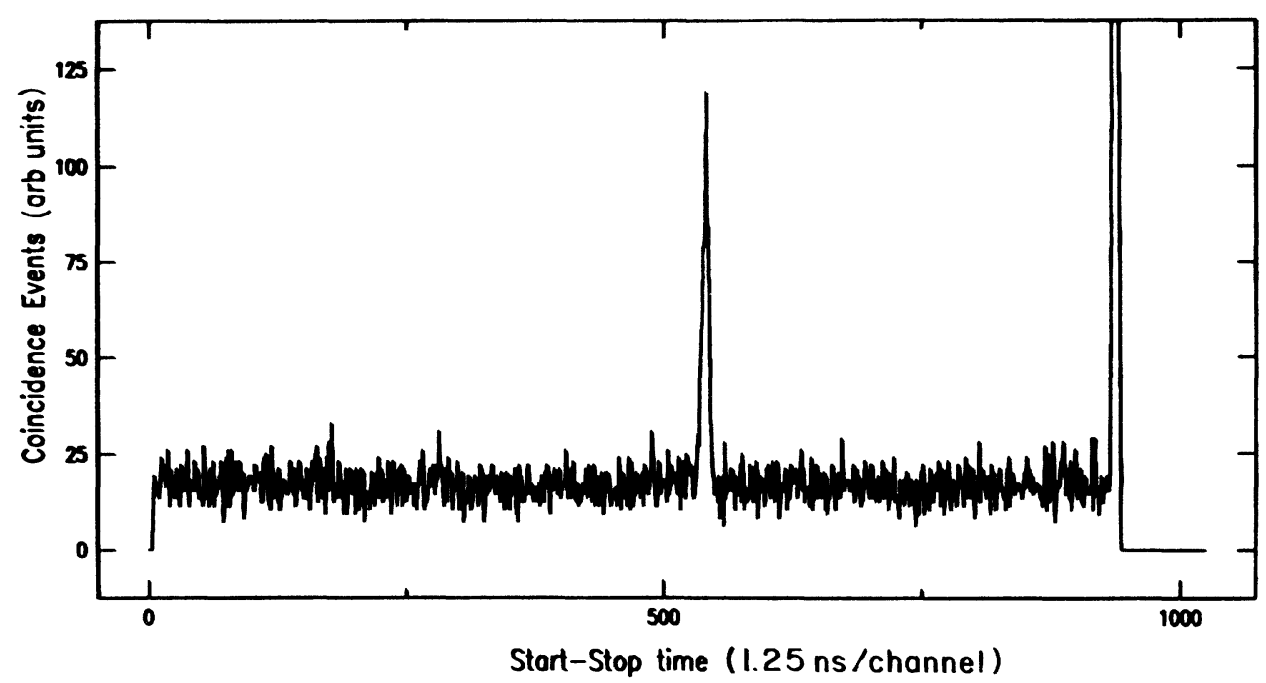

FIG. 3. Coincidence time spectrum taken at an angle of $0.1 \mathrm{mrad}$ for protons colliding with Ar. The acquisition time for this spectrum was approximately $10 \mathrm{~min}$.

ture cross sections were taken from measured data by Hughes et al. [15]. The change in photon count rate as a function of the target observed in our experiment is consistent with these previously measured data.

In principle, the determination of a $2 p$ capture cross section from the coincidence count rate requires knowledge of the angular distribution of the emitted photons. It has been shown $[21,30]$ that for electric dipole radiation the coincidence count rate $d N / d t$ as a function of the projectile scattering angle $\Theta_{n}$ is related to the differential cross sections for capture to the $2 p_{0}$ and $2 p_{ \pm 1}$ substates $(d \sigma / d \Omega)_{0}$ and $(d \sigma / d \Omega)_{1}$ by

$$
\begin{aligned}
& \frac{d N\left(\Theta_{n}\right)}{d t}=A\left\{7\left(\frac{d \sigma}{d \Omega}\right]_{0}+11\left(\frac{d \sigma}{d \Omega}\right]_{1}-3\left(\frac{d \sigma}{d \Omega}\right)_{1} \sin ^{2} \Theta_{\gamma} \cos \left[2\left(\Phi_{n}-\Phi_{\gamma}\right)\right]\right. \\
&\left.-3\left[\left(\frac{d \sigma}{d \Omega}\right]_{0}-\left[\frac{d \sigma}{d \Omega}\right]_{1}\right] \cos ^{2} \Theta_{\gamma}+3 \sqrt{2} \operatorname{Re}\left\langle a_{0} a_{1}^{*}\right\rangle \sin \left(2 \Theta_{\gamma}\right) \cos \left(\Phi_{n}-\Phi_{\gamma}\right)\right\}
\end{aligned}
$$

where $A$ is a constant of proportionality. The angles $\left(\Phi_{n}, \Theta_{n}\right)$ and $\left(\Phi_{\gamma}, \Theta_{\gamma}\right)$ are the azimuthal and polar angles of the scattered particle and emitted photon with respect to a coordinate system with its $z$ axis in the direction of the incident proton. The detector geometry in our experiment is shown in Fig. 4. The term $\operatorname{Re}\left\langle a_{0} a_{1}^{*}\right\rangle$ is the density-matrix element which describes the relative phase between the $m_{l}=0$ and \pm 1 scattering amplitudes. For $\Phi_{n}=0^{\circ}$ and $\theta_{\gamma}$ and $\Phi_{\gamma}=90^{\circ}$, the photon detector is mounted perpendicular to the scattering plane and Eq. (1) reduces to

$$
\frac{d N\left(\Theta_{n}\right)}{d t}=7 A\left[\left(\frac{d \sigma}{d \Omega}\right)_{0}+2\left(\frac{d \sigma}{d \Omega}\right)_{1}\right] .
$$

Since the collision plane contains the $z$ axis the cross sections for populating the $m_{l}=+1$ and -1 states are equal. The differential $2 p$ capture cross section is therefore given by

$$
\left[\frac{d \sigma}{d \Omega}\right)_{2 p}=\left(\frac{d \sigma}{d \Omega}\right)_{0}+2\left(\frac{d \sigma}{d \Omega}\right)_{1},
$$

and under the conditions leading to Eq. (2) the coin-

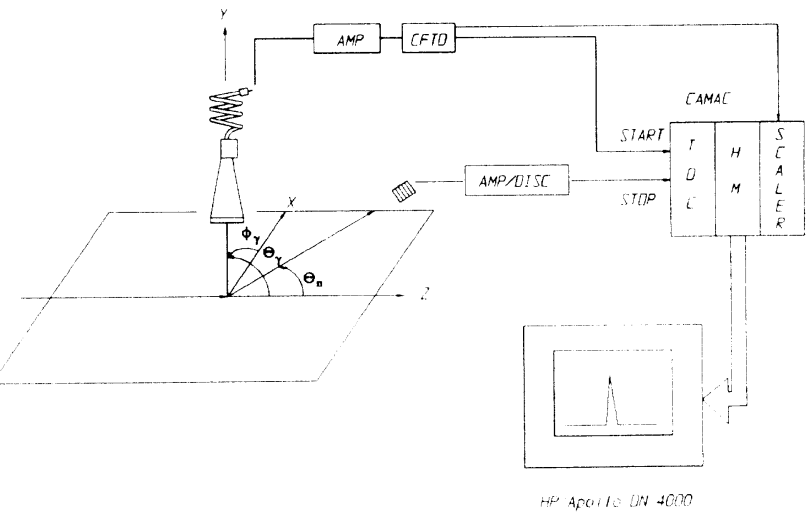

FIG. 4. Detector geometry and electronic coincidence setup. The photon detector was mounted perpendicular to the scattering plane defined by the incident and scattered beam axes. The scattering angle is $\Theta_{n}$, and $\Theta_{\gamma}$ and $\phi_{\gamma}$ are the polar and azimuthal angles of the photon detector with respect to the scattering plane. The acronyms CFTD, TDC, and HM stand for constant-fraction timing discriminator, time-to-digital converter, and histogramming memory, respectively. 
cidence count rate is thus proportional to the differential $2 p$ capture cross section. It should be noted that if any of the photons detected are emitted in a direction not perpendicular to the scattering plane the $2 p$ capture cross section can only be determined if the relative population of the $m_{l}=0$ and \pm 1 states are known. Furthermore, Eq. (2) does not apply to measurements of total cross sections where the scattered projectile is not detected since in that case the collision plane is no longer defined.

In our experiment the photon detector was mounted perpendicular to the scattering plane. However, due to the acceptance angle of the neutral-atom and the photon detectors, some photons were detected which were not emitted perpendicular to the scattering plane. Equation (1) therefore has to be integrated over the acceptance angle of both detectors and then takes the form

$$
\frac{d N\left(\Theta_{n}\right)}{d t}=C\left[\left(\frac{d \sigma}{d \Omega}\right)_{0}+2 D\left(\frac{d \sigma}{d \Omega}\right)_{1}\right],
$$

where $C$ is another constant of proportionality and $D$, which depends on the scattering angle, is a factor resulting from the integration over the finite detector solid angles. The coincidence rate is now only proportional to the differential $2 p$ capture cross section if $D=1$. The values of $D$ calculated for our detector geometry are listed for some scattering angles in Table I. From this table one can see that except for $0^{\circ}, D$ is equal to 1 to within less than $10 \%$ and that the coincidence count rate is thus proportional to the differential $2 p$ capture cross section to a good approximation. In order to estimate the error introduced by the acceptance angles of the detectors, we rewrite (4) as

$$
\frac{d N}{d t}=C\left[\left(\frac{d \sigma}{d \Omega}\right]_{2 p}+2(D-1)\left[\frac{d \sigma}{d \Omega}\right]_{1}\right],
$$

where $(d \sigma / d \Omega)_{0}$ obtained from Eq. (3) was substituted in (4). The second term on the right-hand side can be considered the error introduced by the acceptance angles of the detectors. According to measured data by Hippler et al. [31] $\sigma_{1} / \sigma_{2 p} \approx 0.2$ for the $\mathrm{He}$ target. Assuming that these numbers are similar for the $\mathrm{Ne}$ and Ar targets and that they do not vary significantly with scattering angle, we estimate the relative error to be of the order of $4 \%$ at

TABLE I. Result of the integration of Eq. (1) over the acceptance angles of the projectile and the photon detectors. For $D=1$ the coincidence count rate is proportional to the differential $2 p$ capture cross section.

\begin{tabular}{cl}
\hline \hline $\begin{array}{c}\text { Angle } \\
\text { (mrad) }\end{array}$ & \multicolumn{1}{c}{$D$} \\
\hline 0.0 & 0.79 \\
0.2 & 0.91 \\
0.4 & 0.98 \\
0.6 & 0.99 \\
0.8 & 0.995 \\
1.0 & 0.995 \\
1.5 & 1 \\
2.0 & 1 \\
\hline \hline
\end{tabular}

$0.2 \mathrm{mrad}$. At larger scattering angles the error will even be smaller since the deviation of $D$ from 1 is systematically decreasing with increasing scattering angle.

\section{RESULTS AND DISCUSSION}

In Fig. 5 the differential cross sections for capture with simultaneous emission of a photon are plotted as a function of the laboratory scattering angle for $\mathrm{He}, \mathrm{Ne}$, and Ar, respectively. The error bars show statistical errors only. The systematic errors are mainly determined by the errors of the total $2 p$ capture cross sections to which we normalized our relative differential cross sections integrated over the scattering angle. The errors reported for the measured total $2 p$ capture cross sections are $30 \%$ for $\mathrm{He}$ [29] and 50\% for $\mathrm{Ne}$ and $\mathrm{Ar}$ [15].

While the total $2 p$ capture cross sections depend strongly on the target $\left(1.9 \times 10^{-18} \mathrm{~cm}^{2}\right.$ for $\mathrm{He}$, $3.4 \times 10^{-18} \mathrm{~cm}^{2}$ for $\mathrm{Ne}$, and $1.1 \times 10^{-17} \mathrm{~cm}^{2}$ for Ar), only

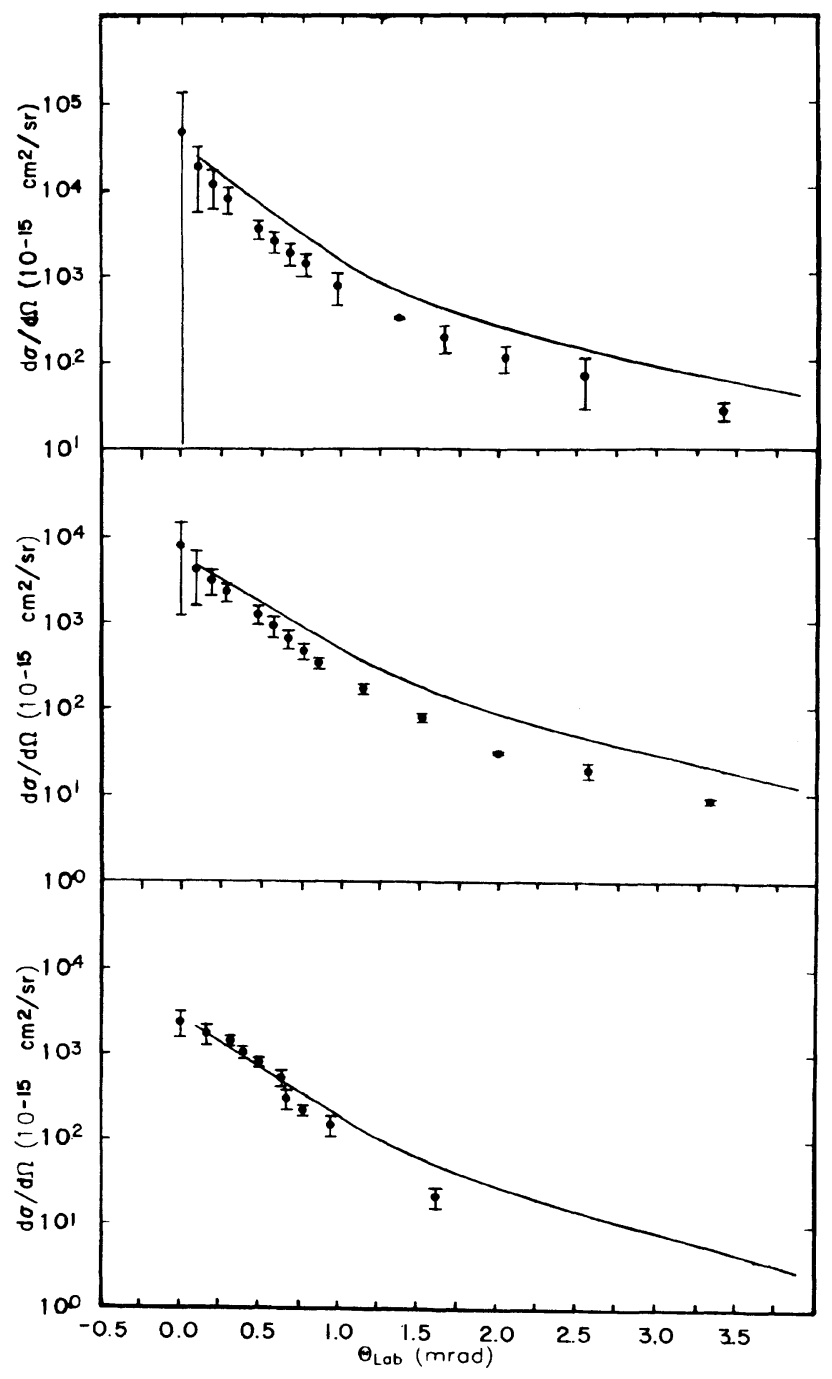

FIG. 5. Differential cross sections in the laboratory frame for capture to the $2 p$ state of hydrogen from Ar (top), Ne (center), and $\mathrm{He}$ (bottom). 
small differences are observed in the shapes of the differential cross sections. There is a noticeable difference only at very small angles where the data for He are somewhat flatter than those for $\mathrm{Ne}$ and Ar. In an attempt to understand the similarity in the shape of the differential cross sections, we have calculated impact-parameterdependent $2 p$ capture probabilities $P(b)$ from the differential cross sections. $P(b)$ is related to the differential cross section by

$$
P(b)=\left(\frac{d \sigma}{d \Omega}\right) \sin \Theta /\left(b \frac{d b}{d \Theta}\right) .
$$

The impact parameter $b$ and $d b / d \theta$ were calculated using a model potential for the target nucleus fit to the Hartree-Fock potential, in which screening is accounted for by a superposition of a number of exponential screening functions [32]. It should be noted that by calculating the impact parameter using only screened nuclear potentials, binary collisions of the projectile with the target electrons are neglected. On the other hand, at large collision distances the scattering angle may be strongly affected by such binary collisions with the target electrons. Here, we use the impact-parameter dependence only to make some qualitative arguments. The $P(b)$ 's ob-

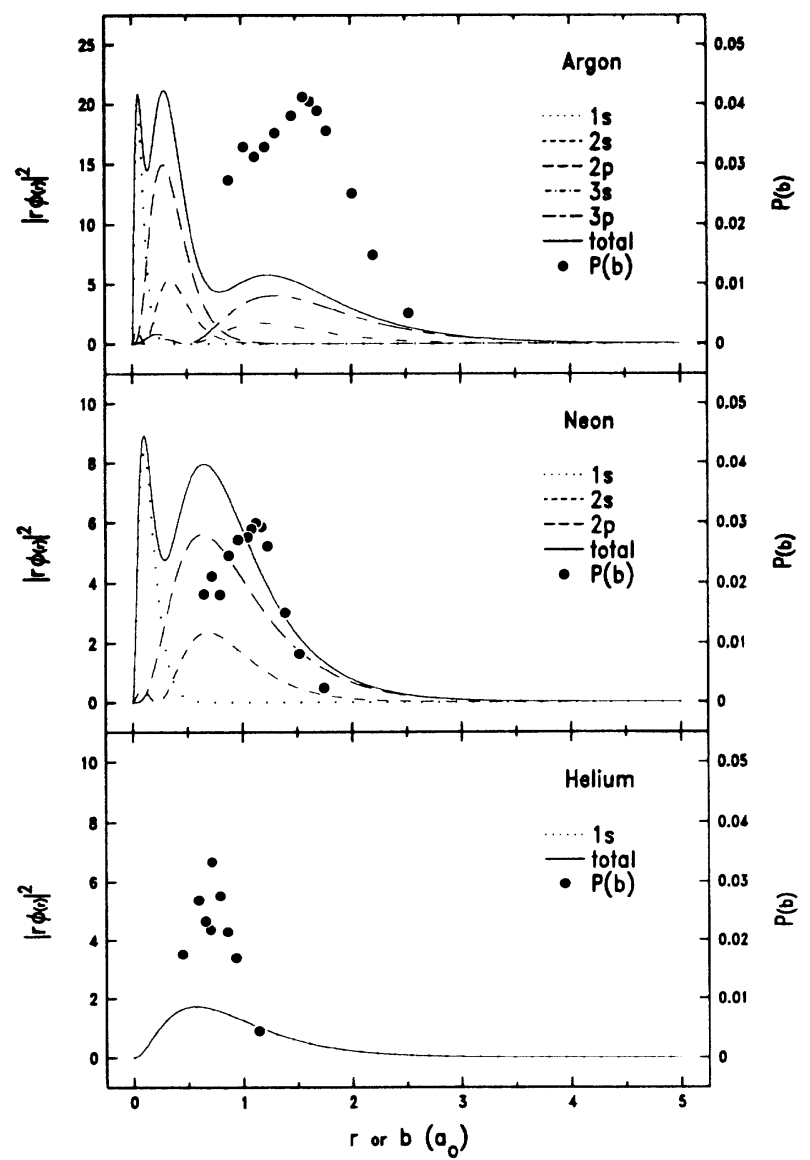

FIG. 6. Impact-parameter-dependent $2 p$ capture probabilities for $\mathrm{Ar}$ (top), $\mathrm{Ne}$ (center), and $\mathrm{He}$ (bottom). Also shown is the radial distribution of the target electrons for various $n l$ states. tained for the three targets using Eq. (6) are shown in Fig. 6. For all targets a pronounced maximum can be seen at relatively large impact parameters. If one extrapolates the small-impact-parameter wing of these maxima to zero-impact parameter, it appears that the $2 p$ capture probability is essentially zero for very close collisions for the $\mathrm{He}$ and $\mathrm{Ne}$ targets and is strongly reduced for the $\mathrm{Ar}$ target compared to far collisions.

At a given impact parameter, the projectile predominantly penetrates the electron cloud for a certain $n$ state. A comparison of the impact-parameter-dependent probabilities with the spatial distribution of the electrons of a given $n$ state may therefore give some information about the $n$ state an electron is captured from. The curves in Fig. 6 show the square of the product of the radial wave function with the radius $|r \phi(r)|^{2}$ for the individual $n l$ terms of the ground state and for their sum. For the wave functions the parametrized representation given by Clementi and Roetti [33] was used. It can be seen that for all targets the maximum in $P(b)$ is close to the maximum in $|r \phi(r)|^{2}$ for the valence electrons and that $P(b)$ is small in the region where $|r \phi(r)|^{2}$ maximizes for the

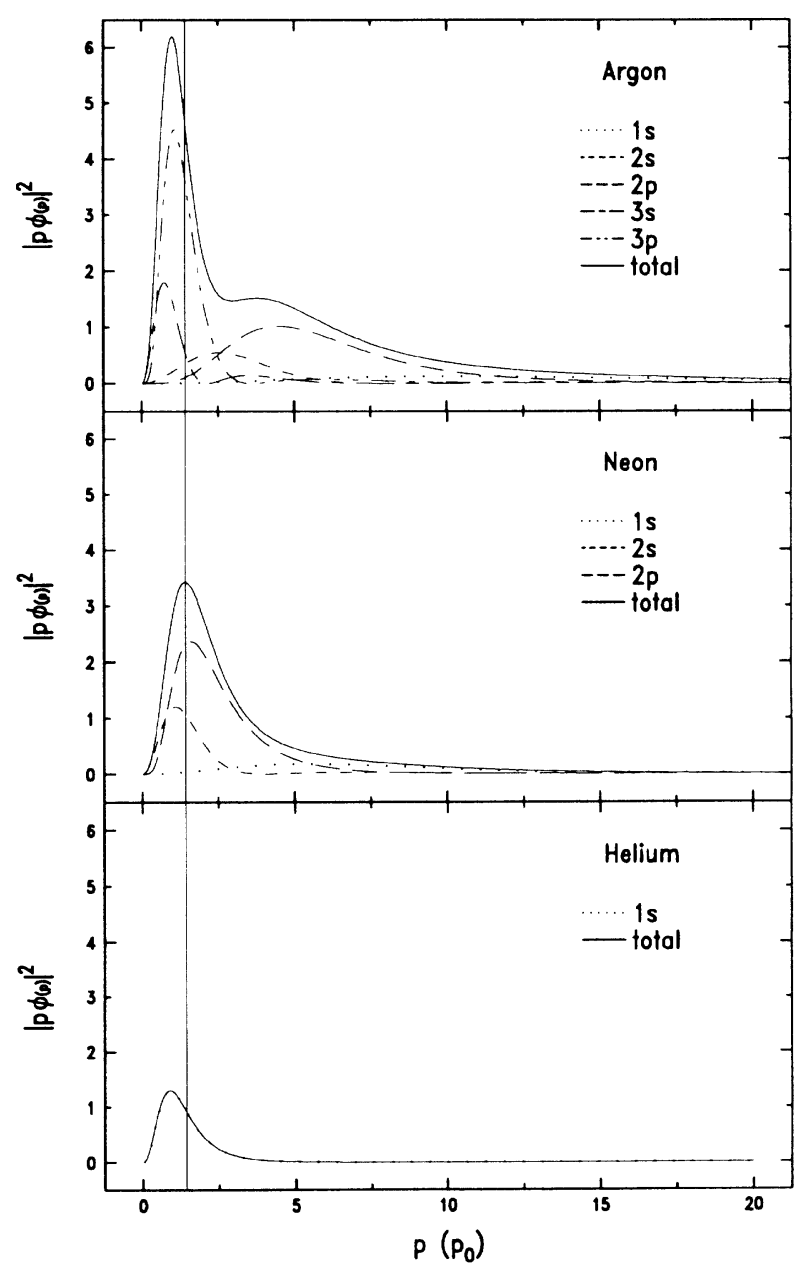

FIG. 7. Momentum distribution of the target electrons for $\mathrm{Ar}$ (top), $\mathrm{Ne}$ (center), and $\mathrm{He}$ (bottom). The vertical line indicates the reduced projectile momentum, which is the electron mass times the proton velocity. 
inner shells. In the case of the Ar target there may be some contributions to $P(b)$ when the $L$ shell or even the $K$ shell is penetrated if the data are extrapolated to small-impact parameters.

From the radial wave functions alone one cannot understand why the $P(b)$ 's are strongly reduced for close collisions where the inner shells are penetrated. However, a capture process does not only require a spatial overlap between the projectile and the target electron, but also an overlap of the projectile velocity with the velocity distribution of the target electrons. In Fig. 7 the square of the product of the electron momentum with the momentum wave function, which is simply the Fourier transform of the radial wave function, is plotted versus the electron momentum. The vertical line indicates the reduced projectile momentum (projectile velocity times electron mass). Again, as for the various radial wave functions, there is a strong overlap in momentum space with the valence electrons and almost zero overlap with the inner shells. Only the Ar target has a small overlap of the reduced projectile momentum with the momentum distribution of the $2 s$ state. This lack of overlap in momentum space with the inner shells, which are only penetrated in close collisions, is a possible explanation for the small $2 p$ capture probabilities at small-impact parameters. Also, the nonzero overlap with the $2 s$ state of Ar may explain why the extrapolated probabilities do not fall as quickly at small-impact parameters for the Ar target as they do with the $\mathrm{He}$ and $\mathrm{Ne}$ targets. We are therefore led to conclude that capture to the $2 p$ state is dominated by the valence electrons of the target with possibly some small contributions from the $2 s$ state for the Ar target.

The full curves in Fig. 5 show a classical-trajectory Monte Carlo calculation by Olson and Lundy [34] for $\mathrm{Ne}$ and Ar and by Schultz et al. [35] for He. There are currently no other calculations available to the best of our knowledge. The CTMC technique has been described in detail by, e.g., Olson and Salop [36]. In this calculation only capture from the valence shell of the target atom was taken into account, which is consistent with our conclusion above from our data. For the He target the agreement between the calculation and the data is excellent both in absolute magnitude and in the angular shape. Only at the largest measured scattering angle is there significant discrepancy. For the $\mathrm{Ne}$ and the Ar target there is good agreement with the data in the angular shape. Even though in absolute magnitude the calculation is somewhat higher in both cases the discrepancy is within the systematic error in the data.

\section{CONCLUSIONS}

We have studied capture to the $2 p$ state of hydrogen for protons colliding with $\mathrm{He}, \mathrm{Ne}$, and $\mathrm{Ar}$ targets at a projectile energy of $50 \mathrm{keV}$ by measuring coincidences between neutralized projectiles and Lyman- $\alpha$ photons emitted by the hydrogen atoms. While the total cross sections depend strongly on the target the angular distribution of the differential cross sections do not differ significantly for the targets studied here. From a comparison of the impact-parameter-dependent probabilities extracted from the measured differential cross sections with the spatial distribution of the target electrons we conclude that capture to the $2 p$ state is dominated by the valence electrons of the target for the collision systems studied here. The small overlap of the reduced projectile momentum with the momentum distribution of the inner-shell target electrons explains why capture from an inner shell is unlikely even for a close collision. The dominance of the valence electrons in $2 p$ capture, in turn, explains the similarity in the angular distribution of the differential cross sections for different target $Z$ 's. The very good agreement of our data with a calculation which only takes capture from the valence shell into account supports our conclusion.

\section{ACKNOWLEDGMENTS}

We would like to thank Dr. R. E. Olson for making his calculations available to us prior to publication. This work was supported by the National Science Foundation.
[1] E. Rutherford, Philos. Mag. 47, 277 (1924).

[2] J. R. Oppenheimer, Phys. Rev. 31, 349 (1928).

[3] H. C. Brinkmann and H. A. Kramers, Proc. Acad. Sci. Amsterdam 33, 973 (1930).

[4] N. Bohr and J. Lindhard, K. Dan. Vidensk. Selsk. Mat. Fys. Medd. 28, No. 7 (1954), and references therein.

[5] H. B. Gilbody, Adv. At. Mol. Phys. 15, 293 (1979).

[6] R. E. Olson and A. S. Schlachter, Comments At. Mol. Phys. 18, 237 (1986).

[7] R. A. Mapleton, Theory of Charge Exchange (WileyInterscience, New York, 1972).

[8] M. Kimura and C. D. Lin, Phys. Rev. A 34, 176 (1986).

[9] M. Kimura and N. F. Lane, Advances in Atomic and Molecular Physics, edited by D. Bates and B. Bederson (Academic, New York, 1990), Vol. 26, p. 79.

[10] B. H. Bransden and D. P. Dewangan, Advances in Atomic and Molecular Physics, Vol. 25, p. 343.
[11] C. Havener, in Physics of Electronic and Atomic Collisions, edited by W. R. MacGillivray, I. E. McCarthy, and M. C. Standage (Hilger, Bristol, 1992), p. 381.

[12] W. Schwab, G. B. Baptista, E. Justiniano, R. Schuch, H. Vogt, and E. W. Weber, J. Phys. B 20, 2825 (1987).

[13] C. Schmeissner, C. L. Cocke, R. Mann, and W. Meyerhoff, Phys. Rev. A 30, 1661 (1984).

[14] C. Biedermann et al., Phys. Rev. A 43, 4075 (1991).

[15] R. H. Hughes, E. D. Stokes, Song-Sik Choe, and T. J. King, Phys. Rev. A 4, 1453 (1971).

[16] M. B. Shah, J. Geddes, and H. B. Gilbody, J. Phys. B 13, 4049 (1980).

[17] P. J. Martin, K. Arnett, D. M. Blankenship, T. J. Kvale, J. L. Peacher, E. Redd, V. C. Sutcliffe, J. T. Park, C. D. Lin, and J. H. McGuire, Phys. Rev. A 23, 2858 (1981).

[18] T. R. Bratton, C. L. Cocke, and J. R. Macdonald, J. Phys. B 10, L517 (1977). 
[19] E. Horsdal-Pedersen, C. L. Cocke, and M. Stöckli, Phys. Rev. Lett. 50, 1910 (1983).

[20] H. Vogt, R. Schuch, E. Justiniano, M. Schulz, and W. Schwab, Phys. Rev. Lett. 57, 2256 (1986).

[21] R. H. McKnight and D. H. Jaecks, Phys. Rev. A 4, 2281 (1971).

[22] R. Hippler, M. Faust, R. Wolf, H. Kleinpoppen, and H. O. Lutz, Phys. Rev. A 31, 1399 (1985).

[23] R. Hippler, M. Faust, R. Wolf, H. Kleinpoppen, and H. O. Lutz, Phys. Rev. A 36, 4644 (1987).

[24] D. G. Seely, S. W. Bross, A. D. Gaus, J. W. Edwards, D. R. Schultz, T. J. Gay, J. T. Park, and J. L. Peacher, Phys. Rev. A 45, R 1287 (1992).

[25] J. T. Park, Advances in Atomic and Molecular Physics, edited by D. Bates and B. Bederson (Academic, New York, 1983), Vol. 19, p. 67.

[26] S. R. Walther, K. N. Leung, and W. B. Kunkel, Rev. Sci.
Instrum. 57, 1531 (1986).

[27] J. T. Park, J. M. George, J. L. Peacher, and J. E. Aldag, Phys. Rev. A 18, 48 (1978).

[28] E. Redd and J. T. Park, Rev. Sci. Instrum. 55, 119 (1984).

[29] Oak Ridge National Laboratory Report No. 6086/V1, 1990 (unpublished).

[30] J. Macek and D. H. Jaecks, Phys. Rev. A 4, 2288 (1971).

[31] R. Hippler, W. Harbich, M. Faust, H. O. Lutz, and L. J. Dubé, J. Phys. B 19, 1507 (1986).

[32] H. L. Cox and R. A. Bonham, J. Chem. Phys. 47, 2599 (1967).

[33] E. Clementi and C. Roetti, At. Data Nucl. Data Tables 14, 177 (1974).

[34] R. E. Olson and C. Lundy (private communication).

[35] D. R. Schultz, C. O. Reinhold, R. E. Olson, and D. G. Seely, Phys. Rev. A 46, 275 (1992).

[36] R. E. Olson and A. Salop, Phys. Rev. A 16, 531 (1977). 